\title{
VARIETIES AND FINITE GROUPS ${ }^{1}$
}

\author{
L. G. KOVÁCS
}

(Received 8 December 1967)

The theory of discrete, abstract groups, as presented in current texts, consists of investigations of various special classes of groups: it has very few completely general results. For some classes (say, for finite groups) the investigations have been extensive and successful; in a few cases (say, for finitely generated abelian groups) they have even reached a sense of completeness. The choice of some of these classes was dictated by the needs of other branches of mathematics. Many more were introduced with the view of extending the scope of certain powerful but special results, and a large part of the literature is taken up by elaborate counterexamples which mark the limits of these generalizations. In so far as one is looking for some kind of classification theory, it is immediately evident that the classes investigated were chosen by historical accident rather than by any master plan, and so far do not appear to form the initial part of a pattern which could be enlarged and completed in the future.

Into this unsatisfactory picture of the classification problem of group theory, varieties bring a ray of hope. These are classes of groups selected by a single principle. Their study offers a reasonably balanced approach: this classification is fine enough not to become trivial even if one thinks only of finite groups, and yet there are not so many classes as to escape a manageable, although again non-trivial, pattern. While not all formerly considered classes are varieties, many of the important ones can at least be approximated by varieties. The successes of this young theory leave little doubt that there is a great deal more to come.

The theory is young. It started exactly 30 years ago, with a paper of B. H. Neumann [12]. It has grown spectacularly during the last 15 years, and its present state has been summed up in Hanna Neumann's monograph [15] published three months ago. ${ }^{2}$ The occasion seems right to give some of our attention to the achievements and to ponder possible directions of progress.

1 This is an expanded version of an invited address given on 2nd June, 1967, at the Annual Meeting of the Australian Mathematical Society, at Canberra.

2 There are also two survey papers about to appear: one by Hanna Neumann [14] and one by B. H. Neuman [13]. 
We need at least a little technical detail. If $g, h$ are elements of a group $G$ and

$$
g^{-1} h g^{2} h^{-1} g^{-1}=1,
$$

one says that $g, h$ satisfy the relation (1). This common phrase indicates that it is possible to conceive of the relation independent of $g, h$, and that another pair $g^{\prime}, h^{\prime}$ of elements of $G$ may or may not satisfy it. Formally, this can be put as follows. The relation, or rather the relator corresponding to its left hand side, is to be an element of a free group $X$ freely generated by (infinitely many) 'variables' $x_{1}, x_{2}, \cdots$; in this case, we may take

$$
x_{1}^{-1} x_{2} x_{1}^{2} x_{2}^{-1} x_{1}^{-1} \text {. }
$$

The claim that $g, h$ satisfy (1) means that the image of (2) is the identity element 1 of $G$ under every homomorphism of $X$ to $G$ which maps $x_{1}$ to $g$ and $x_{2}$ to $h$. If every pair of elements of $G$ satisfies (1), then (2) is called an identical relator, or law, of $G$. Thus

$$
\cap\{\operatorname{ker} \phi \mid \phi: X \rightarrow G\}
$$

is the set of all laws of $G$. This set is an isomorphism-invariant of $G$, and it certainly contains a lot of essential information about $G$; to mention an extreme case, if two finite simple groups have precisely the same laws, they must be isomorphic. ${ }^{3}$

A variety can be defined from an arbitrary subset $L$ of $X$ as the class of those groups in which every element of $L$ is a law. It is obvious that a variety contains all subgroups, all homomorphic images, and all cartesian products of its members. Conversely, it can be shown that every class of groups which has this closure property is a variety. Different subsets of $X$ can define the same variety, but there is a unique largest subset defining any given variety (namely, the subset consisting of all common laws of the groups in the variety). These subsets are precisely the fully invariant subgroups of $X$ : the subgroups which are mapped into themselves by every endomorphism of $X$. Thus there is a one-to-one correspondence between varieties and fully invariant subgroups of $X$. It can, therefore, be stated that the number of varieties is some cardinal between $\boldsymbol{\aleph}_{0}$ and $2^{\boldsymbol{\aleph}_{0}}$ (inclusive); the precise number is one of the great unknowns. It would, of course, be $\boldsymbol{\aleph}_{0}$ if every variety could be defined from a finite subset of $X$, but no-one knows whether this is the case: we have arrived at the so-called finite basis problem. Partial solutions have been given, perhaps the deepest by Sheila Oates (now Mrs I. D. Macdonald) and M. B. Powell. They proved

${ }^{3}$ I am not sure who was the first to observe this (non-trivial) fact; it occurs as item 53.35 in Hanna Neumann's book [15]. In the rest of this lecture, I shall not give detailed references for results which can be found in [15]. 
that if $G$ is any finite group, then the variety defined by the laws of $G$ can in fact be defined by a finite subset of $X$.

I said that varieties provide a classification which does not reduce to a triviality for the case of finite groups and yet all varieties fit into a reasonably manageable pattern. What is this pattern? First of all, of course, settheoretic inclusion gives a complete, modular lattice order on the set of varieties, with the variety $\mathfrak{D}$ of all groups as maximal element and the variety $[5$ of one-element groups as minimal element. The fact that this lattice is not distributive does not seem to have been recognized until 1965 . The lattice does not satisfy the maximum condition; whether it satisfies the minimum condition is equivalent to the finite basis problem. There is a fairly natural binary operation, called multiplication, on the set of varieties: the product $\mathfrak{U} \mathfrak{B}$ of the varieties $\mathfrak{U}, \mathfrak{B}$ is the class of all extension of groups in $\mathfrak{U}$ by groups in $\mathfrak{B}$ :

$$
\mathfrak{U P}=\{G \mid \exists N \triangleq G . \quad N \in \mathfrak{U} \& G / N \in \mathfrak{B}\} .
$$

Multiplication is compatible with the lattice order; $D$ behaves like zero, and $\mathbb{E}$ is the identity element. A deep and obviously fundamental theorem states that this multiplication turns the set of varieties into a free semigroup (with zero and identity); it was proved, simultaneously and independently, by the three Neumanns (Bernhard, Hanna, and their eldest son Peter) on the one hand and by A. L. Smel'kin on the other.

A few words about methods. As laws are elements of a free group, it is only natural that applications of the theory of free groups (in particular, of commutator calculus) permeate the literature on varieties. The second fundamental tool, to which the theory owes much of its recent flourish, is provided by various wreath products. This construction was the basic idea (of Frobenius) behind monomial and, more generally, induced representations (without which there could be hardly any representation theory), and it has had a most remarkable renaissance since the war, since its applicability to infinite situations has been realized. While outside variety theory many of its products were negative, namely ingenious counterexamples, in our context it produced positive results, including the NeumannsSmel'kin Theorem I just mentioned.

To make a point at the welcome risk of provoking some controversy, I venture to draw a distinction between variety theory and the rest of group theory. Such a dividing line is always hard to draw and impossible to keep rigid; in this case, at least the theory of free groups must be considered a demarkation zone. Beyond that, let us say that variety theory deals with laws and with classes of groups defined with reference to laws, while the rest of group theory deals with arithmetic properties, subgroup structure, various group-theoretic constructions, representations and characters, etc., 
and with classes of groups defined in terms of these concepts. In the sequel, 'group theory' and 'grouptheoretic' will refer only to 'the rest'.

Nobody would dispute that contacts between the two divisions are vitally important both for the internal development of variety theory and for extending its applicability. I would go further, and say that the establishment of a new contact is potentially more valuable than the solution of one or another major problem. For example, the Oxford school of Professor Graham Higman has done a great deal to open up an interplay between variety theory and the very rich theory of finite groups. The fact that they have made some of the powerful methods of finite group theory usable in variety theory, seems to me even more important than the striking results they have obtained so far. Towards the end I shall mention the one occasion when benefits went the other way, in that varietal methods contributed to an impressive result in finite group theory.

I turn now to some explicit problems and results. They are selected for illustration, and the selection reflects my interests rather than any value-judgement. Most of them involve finite groups and are, at this stage, unpublished.

The work of Cross, Oates, and Powell, which culminated in the finite basis theorem I mentioned, started with the new concept of critical group. A finite group $G$ is called critical if there exists a variety which contains all proper subgroups and proper factor groups of $G$, but not $G$ itself. A critical group is necessarily monolithic (in the sense that it has only one minimal normal subgroup), but not every monolithic group is critical. One of the cornerstones of the Oates-Powell proof is a much deeper necessary (grouptheoretic) condition for the criticality of a finite group. M. F. Newman and I later gave a series of sufficient conditions. Even earlier, critical p-groups were extensively investigated by P. M. Weichsel. However desirable it would be to have a precise translation of this varietal concept into ordinary grouptheoretic terms, a necessary and sufficient condition of this kind is still lacking. All the same, even the existing results have proved useful far beyond the finite basis context.

Every locally finite variety (that is, a variety consisting of locally finite groups) is completely determined by the critical groups it contains. Given two locally finite varieties and their critical groups, the join (in the lattice of varieties) of the two varieties is again locally finite, but the determination of the critical groups of the join from the critical groups of the two varieties is usually a very elusive problem. The good extreme is the case of varieties of $s \mathfrak{Y}$-groups (that is, varieties which consist of locally finite groups whose Sylow subgroups are all abelian). P. J. Cossey has proved, essentially in his thesis [1], that the set of critical groups of the join of two such varieties is simply the (set-theoretic) join of the sets of critical groups 
of the component varieties. This result rests partly on the fact that a finite $s \mathfrak{A}$-group is critical if and only if it is monolithic; it led Cossey to the following attractive theory. Let $\mathbb{E}$ be the set of critical sश-groups (considered up to isomorphism). A partial order $\prec$ is introduced in $\mathbb{C}$ by defining $G \prec H$ if $G$ is a homomorphic image of a subgroup of $H$. A subset $\mathfrak{Q}$ of $\mathbb{C}$ is an ideal if $G \prec H \in \mathfrak{L}$ implies $G \in \mathfrak{L}$. An ideal is small if it contains only finitely many cyclic groups. Set-theoretic joins and intersections of (finitely many) small ideals are obviously small ideals; thus the small ideals of $\mathbb{E}$ form a lattice $\mathscr{L}$. Since this is a sublattice of the lattice of all subsets of $\mathbb{E}$, it has many desirable properties, for instance, it is distributive. Now the full set of critical groups of a variety of $s \mathfrak{A}$-groups is a small ideal in $\mathfrak{C}$, and every small ideal occurs precisely once as such. This one-one correspondence between varieties of $s \mathfrak{H}$-groups and elements of $\mathscr{L}$ is a lattice isomorphism; hence the varieties of $s \mathfrak{U}$-groups from a lattice $\mathscr{L}^{*}$ with all the properties of $\mathscr{L}$. In particular, $\mathscr{L}^{*}$ is distributive. To express another good property, call a variety a Cross-variety if it can be defined by the laws of a single finite group. A variety of $s \mathfrak{U}$-groups is Cross if and only if the corresponding small ideal of $\mathbb{E}$ is finite. Therefore each Cross variety of $s \mathfrak{U}$-groups can be written in precisely one irredundant way as the join of join-irreducible varieties: namely, as the join of its maximal join-irreducible subvarieties. The strength of this theory is best offset by the contrast of other cases which one might expect to be well-behaved: say, take the case of varieties consisting of nilpotent groups of class at most 6 and exponent dividing 7. For these, no such results can hold: while there are only finitely many of them and they are all Cross-varieties, their lattice is non-distributive, and some of them can be written in more than one irredundant way as joins of joinirreducible varieties (Graham Higman [5]).

Let us look at locally finite varieties in general. For a start, I have to mention some extreme examples. Kostrikin's famous theorem on the restricted Burnside problem is equivalent to the following statement: for any prime $p$, the class $\Re_{p}$ of all locally finite groups of exponent dividing $p$ is a variety. In this statement $p$ can be replaced by any square-free integer $e$ : for, it follows from a series of results the latest of which have recently been announced by J. H. Walter [16], [17], that to each such integer there are only finitely many finite simple groups of exponent $e$, and so the HallHigman reduction theorems [3] apply. On the other hand, the negative solution of the unrestricted Burnside problem (Novikov, Adyan, Britton) means that $\Re_{e}$ is rarely equal to the variety $\Re_{e}^{(1)}$ defined by the one-variable laws of $\Re_{e}$. Let $\Re_{e}^{(n)}$ denote the variety defined by the (at most) $n$-variable laws of $\AA_{e}$. If it could be shown that there is a square-free $e$ such that $\Re_{e}^{(n)} \neq \Re_{e}$ for every choice of $n$, we would, of course, have a negative solution of the finite basis problem. The complexity of the arguments for $n=1$ 
makes one doubt the feasibility of this approach, but it would be desirable to have the opinion of someone who is really familiar with the difficulties involved.

If $\mathfrak{U}^{(n)}$ is the variety defined by the $n$-variable laws of a locally finite variety $\mathfrak{u}$, then $\mathfrak{u}^{(n)}$ can certainly be defined from a finite subset of $X$; hence the finite basis problem for $\mathfrak{U}$ is in fact equivalent to the question whether $\mathfrak{U}^{(n)}=\mathfrak{U}$ for some $n$. For locally finite $\mathfrak{U}$ in general (although not for $\left.\mathfrak{U}=\mathfrak{R}_{e}\right)$, one is less ambitious in asking whether $\mathfrak{U}^{(n)}$ is locally finite for some value of $n$. If a case were found when the answer is affirmative (as it is whenever $\mathfrak{U}$ is soluble) but $\mathfrak{U}$ itself cannot be defined from a finite subset of $X$, we would still have the answer to the question of what the number of varieties is: namely, in this case already $\mathfrak{U}^{(n)}$ would have $2^{N_{0}}$ distinct subvarieties [8].

One road to the finite basis result of Oates and Powell can be described as follows. Let $\mathfrak{U}$ be the variety defined by the laws of a finite group $G$. For positive integers $e, m, c$, consider the class $\mathfrak{B}$ of those groups which have (i) exponent dividing $e$, (ii) no chief factor of order greater than $m$, and (iii) no nilpotent factor (that is, factor group of a subgroup) of class greater than $c$. If $e, m, c$ are suitably chosen, then $\mathfrak{U} \subseteq \mathfrak{B}$. In any case, $\mathfrak{B}$ can be shown to be a locally finite variety defined from a finite subset $L$ of $X$ and containing only finitely many (isomorphism classes of) critical groups. All that remains is to choose, to each critical group $H$ which lies in $\mathfrak{B}$ but not in $\mathfrak{U}$, a law of $G$ not satisfied by $H$; the finite set obtained by adjoining these laws to $L$ will define $\mathfrak{U}$.

Could one do something like this for other locally finite varieties $\mathfrak{U}$ ? As a first step, one looks for locally finite varieties $\mathfrak{B}$ which contain $\mathfrak{U}$ and which can be defined by conditions like (i-iii) above. To formulate a result in this direction, let us say that a group is simply monolithic if the intersection of its non-trivial normal subgroups is a non-abelian simple group. The class $\mathfrak{B}$ of those groups whose nilpotent factors and finitely generated simply monolithic factors all lie in $\mathfrak{U}$ is always a locally finite variety (containing $\mathfrak{H}$ ) $[7]$. (The proof of this rests on the argument behind the Hall-Higman reduction theorems [3]. It follows from the results of Walter and others mentioned before, that to each positive integer $e$ one can choose a locally finite variety $\mathfrak{U}$ such that the corresponding variety $\mathfrak{B}$ is just the class of all sâf-groups of exponent dividing e.) One can play a different variation if it is assumed that $\mathfrak{U}$ contains only finitely many finite simple groups. (In fact, a somewhat weaker condition is sufficient; but even this does not appear too stringent a restriction if one notes that no locally finite variety can contain infinitely many of the finite simple groups which are known at this stage.) Under this assumption, the class $\mathfrak{W}$ of those groups which have no nilpotent factor outside $\mathfrak{H}$, and have a com- 
position system (in the sense of Kurosh [11]) whose factors all lie in $\mathfrak{U}$, is a locally finite variety (containing $\mathfrak{U}$ ). However, the question remains: what further restriction must be imposed on $\mathfrak{U}$ to make it provable that $\mathfrak{B}$, or some variant of it, can be defined by a finite set of laws? As I mentioned, all is well if $\mathfrak{U}$ is soluble; what happens if $\mathfrak{U}$ is only assumed locally soluble? This condition is too weak to exclude the unmanageable case $\mathfrak{U}=\mathfrak{\Re}_{p}$, for $\Re_{p}$ is even locally nilpotent. In any case, the locally nilpotent groups in $\mathfrak{U}$ form a subvariety $\mathfrak{U}_{L N}$; the best $I$ have been able to do so far is to show that if $\mathfrak{U}_{L N}$ can be defined from a finite set of laws (and $\mathfrak{U}$ is locally soluble), then so can $\mathfrak{B}$. (The proofs of these results will be sketched in Appendix 1 at the end of this paper.)

We have passed close to an intriguing problem: can locally finite varieties contain infinite simple groups? All the locally finite, infinite simple groups I know are excluded on the obvious ground that they do not have finite exponent. (They are the finitary alternating groups on infinite sets, and similar 'finitary but infinite' analogues of other known finite simple groups.) Moreover, each of these groups has arbitrarily large finite factors isomorphic to known non-abelian simple groups and so, by a result of Hermann Heineken and Peter M. Neumann ([4], p. 45) none of them can lie in any variety smaller that $\mathbb{D}$. According to a sharpened form of a theorem of M. I. Kargapolov [6] (see Appendix 2 at the end of this paper), the general situation resembles this at least to the extent that if $G$ is a locally finite, infinite simple group, and $A / B$ is any finite simple factor of $G$, then $G$ has a larger finite simple factor which has a (proper) factor isomorphic to $A / B$. There are some classical, but so far intractable, conjectures on finite simple groups, any one of which would imply that no locally finite variety can contain infinitely many finite simple groups, and hence that the answer to the question is negative.

Finally, let us turn to the one result which I would class as being undisputably an application of varietal methods to a non-varietal problem. It is an old conjecture that every finite simple group can be generated by two elements, but so far the only evidence for it is empirical: there is no grouptheoretic argument which would give anything like this. However, M. B. Powell showed recently, by a very short and ingenious varietal argument, that each of a large class of finite simple groups can be generated by three elements. A finite simple group $G$ belongs to Powell's class if the set of prime divisors of the order of $G$ can be partitioned into two (nonempty) parts such that the order of each proper simple factor of $G$ has all its prime divisors in one or the other part. This class does not include all known finite simple groups, but there is no reason to believe that it is contained in the class of known finite simple groups (unless one believes that all finite simple groups are already known). 
The incredibly powerful current investigations on finite simple groups impress, among other things, by the fact that they use all of finite group theory, including all available information about the detailed structure and representation theory of the known finite simple groups. However, virtually no varietal information is available on these groups. One is led to wonder whether a detailed investigation of the laws of the known finite simple groups might not result in variety theory becoming an additional weapon in this arsenal, perhaps the new weapon needed to simplify some of the marathon proofs and to get past points where present methods fail.

\section{Appendix 1}

For notation, terminology, and standard results, refer to Hanna Neumann's book [15].

Let $\mathfrak{U}$ be a locally finite variety. Recall from pp 6, 7 the definitions: $\mathfrak{u}_{L N}$ is the variety consisting of the locally nilpotent groups in $\mathfrak{U}$. The class of those groups which have no nilpotent or finitely generated simply monolithic factors outside $\mathfrak{U}$, is denoted by $\mathfrak{B}$. The class $\mathfrak{M}$ consists of the groups which have no nilpotent factors outside $\mathfrak{u}$ and have at least one composition system whose factors are all in $\mathfrak{U}$. Define two further classes: $\mathfrak{W}_{1}$ is to be the class of those groups whose nilpotent factors and simple factors all belong to $\mathfrak{U}$, and $\mathfrak{W}_{2}$ the class of those groups whose nilpotent factors and finitely generated simple factors all belong to $\mathfrak{U}$.

The proofs of the following results will be sketched:

(a) $\mathfrak{B}$ is a locally finite variety contained in some power of $\mathfrak{U}$.

(b) If $\mathfrak{H}$ contains only finitely many finite simple groups, then $\mathfrak{W}_{1}$ is a locally finite variety contained in some power of $\mathfrak{u}$, and $\mathfrak{B}_{1}=\mathfrak{W}_{2}=\mathfrak{W}$.

(c) If $\mathfrak{U}$ is locally soluble and, for some (finite) $m$, the variety $\mathfrak{H}_{L N}{ }^{(m)}$ is locally nilpotent, then, for some $n$, the variety $\mathfrak{B}^{(n)}$ is locally soluble and hence also locally finite. (Since $\mathfrak{U}^{(n)} \leqq \mathfrak{B}^{(n)}$, the same conclusions hold for $\mathfrak{U}^{(n)}$, too.)

(d) If $\mathfrak{U}$ is locally soluble and $\mathfrak{U}_{L N}$ can be defined by a finite set of laws, then $\mathfrak{B}$ is locally soluble and can be defined by a finite set of laws.

The essential part of (a) was announced in [7]; its proof rests on the arguments behind the Hall-Higman reduction theorems [3]. The relevant steps of that argument are recalled below, in a suitably adapted terminology.

A class $\mathfrak{X}$ of groups is said to have the Burnside property if $\mathfrak{X}$ consists of finite groups and, for each positive integer $k, \mathfrak{X}$ contains only finitely many (isomorphism classes of) $k$-generator groups.

(1) If $\mathfrak{X}$ has the Burnside property, so does the class of all finitely generated subdirect products of groups from $\mathfrak{X} ;$ if, moreover, $\mathfrak{X}=\mathrm{s} \mathfrak{X}$, then the latter class is just SDX. 
This is a slightly stronger form of Lemma 4.2 .3 of [3]. Its proof runs as follows: Let $x(k)$ denote the order of the largest $k$-generator groups in $\mathfrak{X}$, and let $G$ be a $k$-generator group with a family $\mathfrak{R}$ of normal subgroups $N$ such that $\cap \mathfrak{R}=1$ and $G / N \in \mathfrak{X}$ whenever $N \in \mathfrak{R}$. As is well known, $G$ has at most $b$ subgroups of index at most $x(k)$, where $b$ is a bound depending only on $k$ and $x(k)$, and hence only on $k$ and $\mathfrak{X}$ (see e.g. M. Hall, Jr. [2]). Since $|G / N| \leqq x(k)$ whenever $N \in \mathfrak{N}$, the class $\mathfrak{R}$ cannot contain more than $b$ distinct elements. Thus $|G|=|G: \cap \mathfrak{l}| \leqq x(k)^{b}$; and $G$ is a subgroup of a finite direct product of groups from $\mathfrak{X}$.

Next, define the product of two classes of groups exactly as this was done for varieties on p. 3. Note that the product of two SD-closed classes of finite groups is always an SD-closed class of finite groups, and that, while multiplication of classes is, in general, not associative, it is associative if all the classes involved are SD-closed classes of finite groups: the proof of these claims is straightforward. Lemma 4.2.2 of [3] asserts:

(2) If $\mathfrak{X}$ and $\mathfrak{Y}$ have the Burnside property, so does $\mathfrak{X} Y$.

Finally, the following statement will suffice here, although it does not quite express the full force of the Hall-Higman reduction arguments (cf. [7]):

(3) If an s-closed class $\mathfrak{X}$ has the Burnside property and $\mathfrak{Y}$ is the class of those finite groups whose nilpotent factors and simply monolithic factors all belong to $\mathfrak{X}$, then $\mathfrak{Y}$ is $\mathrm{s}$-closed and contained in some (finite) power of SD $\mathfrak{X}$; in particular, (1) and (2) imply that $\mathfrak{Y}$ has the Burnside property.

Only an outline of the proof is reproduced here. First, note that QS $\mathfrak{Y}=\mathfrak{Y}$; since the cyclic groups in $\mathfrak{Y}$ all belong to $\mathfrak{X}$ and $\mathfrak{X}$ contains only finitely many cyclic groups, there exists a positive integer $e$ such that every group in $\mathfrak{Y}$ has exponent at most e. Put $\mathfrak{Y}_{d}=\{G \mid G \in \mathfrak{Y}$, $\exp G \leqq d\}$; then $\mathfrak{Y}_{1} \subseteq$ sDX, and $\mathfrak{Y}_{1} \subseteq \mathfrak{Y}_{2} \subseteq \cdots \subseteq \mathfrak{Y}_{e}=\mathfrak{Y}$. The proof consists in showing, by induction on $d$, that $\mathfrak{Y}_{d} \subseteq(\mathrm{SDX})^{n(d)}$ for suitable integers $n(d)$. The initial step has already been noted, with $n(1)=1$. For the inductive step, let $G \in \mathfrak{Y}_{d+1}$ and let $S$ be the greatest soluble normal subgroup of $G$. It follows from Theorem A of [3] that $S$ has a chain of normal subgroups with nilpotent factors, the length of the chain, say $l$, being dependent only on $e$ : thus $S \in(\mathrm{SDX})^{l}$. Now $G / S$ is a subdirect product of monolithic groups, say $H_{i}$, with nonabelian monoliths. Each $H_{i}$ is the extension of a subdirect power of a simply monolithic group by a group whose exponent is smaller than that of $H_{i}$ (cf. the proof of 4.4.1 in [3]): thus $H_{i} \in(\mathrm{SDX}) \mathfrak{Y}_{d} \subseteq(\mathrm{SDX})^{1+n(d)}$. It follows now that $G / S \in(\operatorname{SDX})^{1+n(d)}$, and so $G \in(\operatorname{SDX})^{l+1+n(d)}$ : thus the inductive step is established, with $n(d+1)=l+1+n(d)$.

The next lemma will provide the bridge to varietal arguments. If $\mathfrak{X}$ is any class of groups, $\mathfrak{X}_{0}$ denotes the class of all finitely generated subgroups 
of the groups in $\mathfrak{X}$. Obviously, var $\mathfrak{X}=\operatorname{var} \mathfrak{X}_{\mathbf{0}}$. Further, let $\mathfrak{X}_{N}$ denote the class of nilpotent groups in $\mathfrak{X}_{0}$.

(4) If $\mathfrak{X}_{0}$ has the Burnside property, then var $\mathfrak{X}$ is locally finite, $(\operatorname{var} \mathfrak{X})_{0}=Q \mathrm{QSD}_{0},(\operatorname{var} \mathfrak{X})_{N}=Q \mathrm{SD} \mathfrak{X}_{N}$, and the finite simple groups in var $\mathfrak{X}$ are all in $\mathrm{QX}_{\mathbf{0}}$. Conversely, if var $\mathfrak{X}$ is locally finite, then $\mathfrak{X}_{\mathbf{0}}$ has the Burnside property.

The proof of the first two statements, and of the converse, is just the proof of 15.74 in [15], except that 15.73 has to be used in place of 15.72 . Having established this much, one can argue that if $G$ is any $p$-group in QSD $\mathfrak{X}_{0}$, then $G$ belongs to the QSD-closure of the class of Sylow $p$-subgroups of the groups in $\mathfrak{X}_{0}$. The non-trivial part of the remainder follows from Theorem 4 of $[10]$.

Now, to the PROOF of (a). The first step is to show that $\mathfrak{B}_{0}$ has the Burnside property. Note that $\mathfrak{B}=\mathrm{QS} \mathfrak{B} \supseteqq \mathfrak{B}_{0}=Q \mathfrak{B}_{0}$. Put $\mathfrak{X}=\mathfrak{U}_{0}$; then $\mathfrak{X}$ is s-closed and, according to the converse part of (4), has the Burnside property. If $G \in \mathfrak{B}_{0}$, then every finite factor group of $G$ lies in the class $\mathfrak{Y}$ defined from $\mathfrak{X}$ in (3). By (3) and (1), SD $\mathfrak{Y}$ consists of finite groups and is closed with respect to the formation of finitely generated subdirect products. It follows that if $H$ is the intersection of all normal subgroups of finite index in $G$, then $G / H$ is in SD $\mathfrak{Y}$. Consequently, $G / H$ is finite, and so Schreier's Theorem guarantees that $H$ is finitely generated. Thus, unless $H=1$, $H$ has at least one maximal normal subgroup $N$, say. Then $H / N$ is a finitely generated simple factor of $G$, so it lies in $\mathfrak{U}$ and is, therefore, finite. As $N$ has finite index in $G$, the intersection of its conjugates is a normal sugroup of finite index in $G$, contrary to the choice of $H$. This proves that $H=1$. It follows that $\mathfrak{B}_{\mathbf{0}} \subseteq \mathrm{SD} \mathfrak{Y}$ and so $\mathfrak{B}_{0}$ has the Burnside property.

For the second step of the proof of (a), consider $A \times B$ with $A, B \in \mathfrak{B}_{0}$. It will be shown that $A \times B \in \mathfrak{B}$; then, of course, $A \times B \in \mathfrak{B}_{\mathbf{0}}$. First, check that every $p$-factor of $A \times B$ is in $\mathfrak{u}$. Every $p$-factor of a finite group is a factor of a Sylow $p$-subgroup of the group, and every Sylow $p$-subgroup of $A \times B$ is a direct product of Sylow $p$-subgroups of $A$ and $B$; the latter are in $\mathfrak{U}$, hence so is every $p$-factor of $A \times B$. Every nilpotent factor of $A \times B$ is a direct product of $p$-factors; therefore all these are also in $\mathfrak{u}$. Next, let $H / K$ be a simply monolithic factor of $A \times B$, with monolith $M / K$. If $(H \cap A) K \geqq M$ and $(H \cap B) K \geqq M$, then $M^{\prime} \leqq[(H \cap A) K,(H \cap B) K] \leqq K$ would follow, and so $M / K$ would be abelian. Thus it can be assumed that, say, $(H \cap A) K \geqq M$; then $(H \cap A) K=K$, for every non-trivial normal subgroup of $H / K$ contains $M / K$. Note that $(H \cap A) K=H \cap A K$, and conclude that $H / K=H /(H \cap A K) \cong A H \mid A K$, where, of course, $A H \mid A K$ is isomorphic to a factor of $B$. Consequently, every simply monolithic factor of $A \times B$ is isomorphic to a factor of $A$ or $B$, and hence lies in $\mathfrak{U l}$. This 
completes the proof of the claim that $A \times B \in \mathfrak{B}_{0}$.

The third step begins with the observation that, on account of the second step, $\mathrm{D} \mathfrak{B}_{0}=\mathfrak{B}_{0}$. By (4), the first step implies that (var $\left.\mathfrak{B}\right)_{0}=\mathrm{QSD}_{0}$; hence $(\operatorname{var} \mathfrak{B})_{0}=Q_{S} \mathfrak{B}_{0} \subseteq Q S \mathfrak{B}=\mathfrak{B}$. Since a group belongs to $\mathfrak{B}$ if and only if all its finitely generated subgroups do, this proves that var $\mathfrak{B} \subseteq \mathfrak{B}$. The converse inclusion is trivial; also, var $\mathfrak{B}$ is locally finite, again by (4) and the first step of this proof.

It remains to note that $\mathfrak{B}$ lies in a power of $\mathfrak{U}$ : for, according to the first step, $\mathfrak{B}_{0} \subseteq \mathrm{SD} \mathfrak{Y}$ and, according to (3), $\mathfrak{V}$ lies in a power of $\mathfrak{U}$. This completes the proof of (a).

Proof of (b).

It is now assumed that $\mathfrak{U}$ contains only finitely many finite simple groups. The first thing to note is that, because of Theorem 6 of Kargapolov [6] (see also Appendix 2), all simple groups in $\mathfrak{U}$ are finite. Let $\mathfrak{M}$ be the class of all those simply monolithic groups which belong to some power of $\mathfrak{U}$ and whose nilpotent factors all lie in $\mathfrak{U}$. If $G \in \mathfrak{M}$, the monolith of $G$ is one of the simple groups in $\mathfrak{U}$, and $G$ is a subgroup of the automorphism group of that simple group. Thus $\mathfrak{M}$ is the union of finitely many isomorphism classes of finite groups; and it follows that $\mathfrak{M}$ is contained in some power of $\mathfrak{u}$, and therefore so is the variety $\mathfrak{u}_{1}$ generated by $\mathfrak{U}$ and $\mathfrak{M}$. According to (4), $\mathfrak{U}_{1}$ is locally finite; all the nilpotent or finite simple groups of $\mathfrak{U}_{\mathfrak{1}}$ belong to $\mathfrak{H}$; so that, again by Kargapolov's Theorem, all the simple groups of $\mathfrak{U}_{1}$ are finite (and belong to $\mathfrak{U}$ ). Let $\mathfrak{B}_{\mathfrak{1}}$ be defined from $\mathfrak{U}_{1}$ as $\mathfrak{B}$ was from $\mathfrak{U}$. According to (a), $\mathfrak{B}_{\mathfrak{1}}$ is a locally finite variety contained in some power of $\mathfrak{U}_{1}$ and hence also in some power of $\mathfrak{U}$. In particular, all the nilpotent or simple groups of $\mathfrak{B}_{1}$ belong to $\mathfrak{U}_{1}$ and hence to $\mathfrak{U}$ : thus $\mathfrak{B}_{1} \subseteq \mathfrak{W}_{1}$. Observe that $\mathrm{QSW}_{1}=\mathfrak{W}_{1}$. If $G$ is a simply monolithic group in $\mathfrak{W}_{1}$, its monolith belongs to $\mathfrak{U}$ and is therefore finite; consequently, $G$ is finite. Since all simple factors of $G$ belong to $\mathfrak{U}$, it follows that $G$ belongs to some power of $\mathfrak{U}$; by the definition of $\mathfrak{W}_{1}$, the nilpotent factors of $G$ all belong to $\mathfrak{U}$ : thus $G \in \mathfrak{M} \subseteq \mathfrak{H}_{1}$. This proves that $\mathfrak{W}_{1}=\mathfrak{B}_{1}$.

Next, note that $\mathfrak{W}_{1} \subseteq \mathfrak{W}_{2}=\mathrm{QSW}_{2}$ and $\mathfrak{W}_{1} \subseteq \mathfrak{W}=\mathrm{s} \mathfrak{W}$, and that the finite groups of $\mathfrak{W}_{2}$, as well as those of $\mathfrak{W}$, all belong to $\mathfrak{M}_{1}$. It suffices to show that the finitely generated groups $G$ which lie in $\mathfrak{W}_{2}$ or in $\mathfrak{W}$, all belong to $\mathfrak{W}_{1}$. Let $N$ be the verbal subgroup of $G$ corresponding to the variety $\mathfrak{B}_{1}$. Then $G / N$ is finite and so, by Schreier's Theorem, $N$ is finitely generated. If $N=1$, there is nothing to prove. If $N \neq 1$ and $G \in \mathfrak{M}_{2}$, let $K$ be any maximal normal subgroup of $N$. If $N \neq 1$ and $G \in \mathfrak{W}$, let $\mathbb{C}$ be a composition system of $G$ with all factors in $\mathfrak{A}$ : this system must have a jump $C \triangleleft C^{*}$ such that $C \geqq N \leqq C^{*}$; put $K=C \cap N$. In the first case, $N / K$ belongs to $\mathfrak{U}$ because it is finitely generated and simple; in the second 
case, $N / K$ belongs to $\mathfrak{U}$ because $N / K \cong N C / C \leqq C^{*} / C \in \mathfrak{U}$. In either case, $N / K$ is a finitely generated group in $\mathfrak{U}$ and is, therefore, finite. As $K$ is now known to have finite index in $G$, it has only finitely many conjugates in $G$ : the intersection $D$ of these is a normal subgroup of finite index in $G$. Thus if $G \in \mathfrak{W}_{2}$ then $G / D$ is a finite group in $\mathfrak{W}_{2}$ and hence in $\mathfrak{W}_{1}$ : contrary to the fact that $D$ is properly contained in the verbal subgroup $N$. If $G \in \mathfrak{W}$, then $G / D \in \mathfrak{W}$ follows from the choice of $N, K$, and $D$, which ensure that $G / D$ belongs to some power of $\mathfrak{u}$; as in the first case, a contradiction results. The only alternative is, in either case, that $N=1$, that is, $G \in \mathfrak{B}_{1}$. This completes the proof of (b).

Proof OF (c).

It is now assumed that $\mathfrak{U}$ is locally soluble. In this case, $\mathfrak{B}$ coincides with the variety similarly defined from $\mathfrak{U}_{L N}$ in place of $\mathfrak{U}$ : hence one can, without loss of generality, make the stronger assumption that $\mathfrak{U}$ is locally nilpotent; that is, $\mathfrak{H}_{L N}=\mathfrak{H}$. It follows from (a) that $\mathfrak{B}$ is contained in some power of $\mathfrak{U}$; as $\mathfrak{H}$ is now in a product of locally finite varieties of prime-power exponent, $\mathfrak{B} \leqq \Pi\left(\mathfrak{U}_{i} \mid 1 \leqq i \leqq l\right)$, say, where the $\mathfrak{B}_{i}$ are suitable, not necessarily distinct, prime-power-exponent subvarieties of $\mathfrak{H}$. Put

so that

$$
\mathfrak{B}_{k}=\mathfrak{B} \cap \Pi\left(\mathfrak{U}_{i} \mid l-k<i \leqq l\right),
$$

and

$$
\mathfrak{B}_{\mathbf{1}}=\mathfrak{U}_{\imath} \leqq \mathfrak{U}, \mathfrak{S}_{\imath}=\mathfrak{B},
$$

$$
\mathfrak{B}_{k+1} \leqq \mathfrak{U}_{l-k} \mathfrak{B}_{k} \text { whenever } 1 \leqq k<l \text {. }
$$

Now take up the assumption that $\mathfrak{U}^{(m)}$, and hence $\mathfrak{B}_{\mathfrak{1}}^{(m)}$, is locally nilpotent. The point is to show, by induction on $k$, that $\mathfrak{B}_{k}^{(m+k-1)}$ is locally soluble. The initial step is already available; the inductive step will make use of an idea from the (unpublished) proof of Powell's Theorem. Let $1 \leqq k<l$, let $e$ be the exponent of $\mathfrak{U}_{l-k} \mathfrak{B}_{k}$; let $p$ be the unique prime divisor of the exponent of $\mathfrak{u}_{l-k}$, and $q$ the highest power of $p$ which divides $e$. Assume that $\mathfrak{B}_{k}^{(m+k-1)}$ is locally soluble, and let $\mathfrak{B}_{*}$ be the variety defined by the set

$$
\left\{\left(v x_{m+k}^{e / q}\right)^{q} \mid v \in \mathfrak{B}_{k}\left(X_{m+k-1}\right)\right\}
$$

of laws: note that $\mathfrak{B}_{*}=\mathfrak{B}_{*}^{(m+k)}$. Moreover, $\mathfrak{U}_{l-k} \mathfrak{B}_{k} \leqq \mathfrak{B}_{*}$ : for, the $\mathfrak{B}_{k^{-}}$ subgroup of a group $G$ in $\mathfrak{U}_{l-k} \mathfrak{B}_{k}$ is a normal $p$-subgroup $P$, and any value of $x_{m+k}^{e / q}$ in $G$ is an element of $p$-power order, so that $P$ and this value together still generate a $p$-subgroup, that is, a subgroup of exponent dividing $q$ : therefore, the defining laws of $\mathfrak{B}_{*}$ have no non-trivial value in $G$. This proves that $\mathfrak{V}_{k+1} \leqq \mathfrak{U}_{l-k} \mathfrak{B}_{k} \leqq \mathfrak{B}_{*}$, and so $\mathfrak{B}_{k+1}^{(m+k)} \leqq \mathfrak{B}_{*}^{(m+k)}=\mathfrak{B}_{*}$. Let $H$ be any group in $\mathfrak{B}_{k+1}^{(m+k)}$. The $\mathfrak{B}_{k}^{(m+k-1)}$-subgroup $K$ of $H$ consists of products $v_{1} \cdots v_{t}$ of values $v_{1}, \cdots, v_{t}$ of elements of $\mathfrak{B}_{k}\left(X_{m+k-1}\right)$ : the next step is to show, by induction on $t$, that each such product has order dividing $q$. 
If $t=1$, this is an immediate consequence of the fact that $H \in \mathfrak{B}_{*}$. If $t>1$ and $v_{1} \cdots v_{t-1}$ has order dividing $q$, then $v_{1} \cdots v_{t-1}$ is a value of $x_{m+k}^{e / q}$, and so $H \in \mathfrak{B}_{*}$ again implies that $v_{1} \cdots v_{t}$ has order dividing $q$. Thus $K$ has exponent dividing $q$. Now the $m$-generator subgroups of $K$ belong to $\mathfrak{B}_{k+1}$ and so they are finite; being nilpotent groups in $\mathfrak{B}$, they belong to $\mathfrak{U}$; hence $K$ belongs to the locally nilpotent variety $\mathfrak{u}^{(m)}$. To sum up: $H$ has finite exponent, $K$ is a locally nilpotent normal subgroup of $H$, and $H / K$ is locally soluble (for it belongs to $\mathfrak{B}_{k}^{(m+k-1)}$ ). From this information it is easy to deduce that $H$, and therefore every group in $\mathfrak{B}_{k+1}^{(m+k)}$, is locally soluble. This completes the inductive step, and with it the proof of (c).

Proof of (d).

It is now assumed that $\mathfrak{U}$ is locally soluble and $\mathfrak{u}_{L N}=\mathfrak{U}_{L N}^{(m)}$. As in the proof of (c), it can be assumed without loss of generality that $\mathfrak{U}=\mathfrak{U}_{L N}=\mathfrak{U}^{(m)}$; moreover, according to (c), there exist integers $n$ such that $n \geqq m$ and $\mathfrak{B}^{(n)}$ is locally soluble. Then $\mathfrak{B}^{(n)}$ contains no finitely generated simply monolithic groups, and the $n$-generator subgroups of its nilpotent groups all lie in $\mathfrak{U}$ : thus the nilpotent groups of $\mathfrak{B}^{(n)}$ all lie in $\mathfrak{U}$. This shows that $\mathfrak{B}=\mathfrak{B}^{(n)}$; all that remains to note is that a locally finite variety defined by $n$-variable laws can also be defined by a finite set of laws: cf. the proof of $\mathbf{5 1 . 5 4}$ in [15].

\section{Appendix 2}

In view of the use of Theorem 6 of Kargapolov [6] in this paper, it seems worth including a direct argument which in fact yields the strong form of that theorem mentioned on p. 7. The result is:

ThEOREM. Let $G$ be an infinite, simple, locally finite group, and $H / M$ a finite, simple factor of $G$. Then $H / M$ is isomorphic to a proper factor of some finite, simple factor $K / N$ of $G$.

In fact, $H$ has finite subgroups $A$ such that $A M=H$; and if $A$ is chosen minimal with respect to this property, then $K$ and $N$ can be chosen that $K>A N$ and $A \cap(A \cap M) N=A \cap M$ : then, of course,

$$
H / M \cong A / A \cap M \cong A N /(A \cap M) N \neq K / N .
$$

This proof makes use of a simple lemma, which was noted before Theorem 4 in [10] as a consequence of 4.3 and 4.4 of [9]:

Lemma. The simple groups in a variety generated by a finite group are all finite.

Let $A_{0}$ be the subgroup generated by a complete set of representatives of the cosets of $M$ in $H$; then $A_{0}$ is finite, and $A_{0} M=H$. Let $A$ be minimal among those subgroups of $A_{0}$ which satisfy $A M=H$. As $A / A \cap M \cong H / M$, 
the intersection $A \cap M$ is a maximal normal subgroup of $A$. If $M_{1}$ is a proper normal subgroup of $A$, then $M_{1} M<H$ gives that $M_{1}(A \cap M)<A$, and so $M_{1} \leqq A \cap M$. This means that $A \cap M$ is the only maximal normal subgroup of $A$.

According to the Lemma, $G$ is not contained in the variety generated by $A$; hence $G$ has finitely generated subgroups which are not contained in this variety. Choose one such subgroup; as $G$ is generated by the conjugates of $A$, this subgroup is contained in a subgroup $B$ generated by finitely many conjugates of $A$, and $B$ is not in the variety generated by $A$. In particular, the intersection $D$ of the kernels of all the homomorphisms of $B$ onto factor groups of $A$, is non-trivial. Thus $G$ is generated by the conjugates of $D$, so that $B$ is contained in a subgroup generated by finitely many conjugates of $D$ : in other words, $G$ has a finite subgroup $C$ such that the normal closure $K$ of $D$ in $C$ contains $B$. If all maximal normal subgroups of $K$ contained $D$, so would their intersection, which is proper in $K$, characteristic in $K$ and hence normal in $C$ : this would be contrary to the choice of $K$ as the normal closure of $D$ in $C$. Thus $K$ has a maximal normal subgroup $N$ which does not contain $D$, a fortiori does not contain $B$ and hence does not contain some conjugate $g^{-1} A g$ of $A$ which is part of the given generating set of $B$. By changing from $B, X, K, N$ to their conjugates $g B g^{-1}$ etc. if necessary, it can be assumed that $g=1$ : then $K \geqq B \geqq A \geqq N$. Now $A \cap N$ is a proper normal subgroup of $A$; as such, $A \cap N$ is contained in the unique maximal normal subgroup $A \cap M$ of $A$; so that

$$
A \cap(A \cap M) N=(A \cap M)(A \cap N)=A \cap M .
$$

It remains to exclude the possibility that $A N=K$. If this were the case, one would have from $B \geqq A$ that $B=A(B \cap N)$, so that

$$
B / B \cap N \cong A \mid A \cap B \cap N
$$

would follow. However, this and the choice of $D$ would imply that $N \geqq B \cap N \geqq D$, contrary to the choice of $N$.

\section{References}

[1] P. J. Cossey, 'On varieties of $A$-groups', Ph. D. thesis, Australian National University, Canberra, 1966.

[2] Marshall Hall, Jr., 'Subgroups of finite index in free groups', Canad. J. Math. 1 (1949), $187-190$.

[3] P. Hall and Graham Higman, 'On the $p$-length of $p$-soluble groups and reduction theorems for Burnside's problem', Proc. London Math. Soc. (3) 6 (1956), $1-42$.

[4] Hermann Heineken and Peter M. Neumann, 'Identical relations and decision procedures for groups', J. Austral. Math. Soc. 7 (1967), 39-47. 
[5] Graham Higman, 'Representations of general linear groups and varieties of p-groups', Proc. Internat. Conf. Theory of Groups, Austral. Nat. Univ. Canberra, August 1965, pp. 167-173. Gordon and Breach, New York etc. (1967).

[6] M. I. Kargapolov, 'Locally finite groups possessing normal systems with finite factors', Sibirsk. Mat. Z̆. 2 (1961), 853-873.

[7] L. G. Kovács, 'Varieties and the Hall-Higman paper', Proc. Internat. Conf. Theory of Groups, Austral. Nat. Univ. Canberra, August 1965, pp. 217-219. Gordon and Breach, New York etc. (1967).

[8] L. G. Kovács, 'On the number of varieties of groups', J. Austral. Math. Soc. 8 (1968), 444-446.

[9] L. G. Kovács and M. F. Newman, 'Cross varieties of groups', Proc. Roy. Soc. London, Ser. A. $292(1966), 530-536$.

[10] L. G. Kovács and M. F. Newman, 'Minimal verbal subgroups', Proc. Cambridge Philos. Soc. $62(1966), 347-350$.

[11] A. G. Kurosh, The Theory of Groups, Vol. II, Chelsea, New York, 1956.

[12] B. H. Neumann, 'Identical relations in groups, I', Math. Ann. 114 (1937), 506-525.

[13] B. H. Neumann, 'Varieties of groups', Bull. Amer. Math. Soc. 73 (1967), 603-613.

[14] Hanna Neuman, 'Varieties of groups', Proc. Internat. Conf. Theory of Groups, Austral. Nat. Univ. Canberra, August 1965, pp. 251-259. Gordon and Breach, New York, etc. (1967).

[15] Hanna Neumann, Varieties of Groups, Springer, Berlin etc., 1967.

[16 [ J. H. Walter, 'Finite groups with abelian Sylow 2-subgroups of order 8', Invent. Math. 2 (1967), $332-376$.

[17] J. H. Walter, 'The characterization of finite groups with abelian Sylow 2-subgroups', (to appear).

\section{Australian National University}

Canberra 